\title{
Mathematical modelling of the quenching process of 6061 aluminium alloy plates
}

\author{
Marin Petre ${ }^{1, *}$, Raluca Efrem $^{2}$, Nicuşor Constantin Drăghici ${ }^{1}$ and Alexandra Valerica \\ Achim $^{1}$ \\ ${ }^{1}$ ALRO, 116 Piteşti Street, Slatina 230104, Romania \\ ${ }^{2}$ Department of Mathematics, University of Craiova, 13 A.I. Cuza, 200585, Romania
}

\begin{abstract}
In recent decades, due to the increase in computing power, mathematical modelling has experienced a fulminant development in almost all areas. The aluminium industry is one of these areas. One of the main processes for improving the properties of certain aluminium alloys is the solution heat treatment and quenching process. The most common quenchant used for aluminium alloys is water. The main advantage of using a water quenchant is that water can provide the rapid quenching. By considering the temperature dependence of the thermo-physical properties, the non-linear thermo-mechanical direct coupled analysis of the quenching process for a 6061 aluminium alloy plate was achieved. The structural stress due to solid thermal effects were studied by using ANSYS finite element software. The quenching rate, which determines the plate deformation after quenching, was estimated and validated on independent equipment for the research of aluminium alloy quenching process. The developed mathematical model serves as a tool by simulation of various scenarios that may occur in the industrial process.
\end{abstract}

\section{Introduction}

Aluminium alloys are solution treated at temperatures generally in the range of 400 to 550 r.C. The successive steps are: heating up to a preset temperature, keeping the material at this temperature for a determined time and cooling it very fast to obtain a super-saturated solid solution [9].

By solution treatment, the concentration of hardening elements including copper, zinc, magnesium, and (or) silicon in the solid solution is maximized. Thus, the solution temperature is usually close to the liquidus temperature of the alloy since the concentration and dissolution rate of the hardening elements increase with temperature [3, 14].

The objective of the quenching process is to cool the material adequately fast so as to prevent unwanted concentration of alloying elements in the grain boundary structure, but, at the same time not cooling faster than necessary to minimize internal stresses, as this may lead to increased distortion or cracking [11]. The role of quenching however is to increase hardness and improve mechanical properties of the material [1]. The main parameters used in the solution heat treatment are the cooling rate, the soaking time and solution temperature.

\footnotetext{
*e-mail: mapetre@alro.ro
} 
While the last two parameters are specified in the standards, the quenching rate must follow the $\mathrm{C}$ shape curve (Time Temperature Transformation) [4].

Generally, two quenching techniques for the hot metals are used, respectively: by immersing the hot metals into a liquid bath or by spraying the hot metal with a liquid. The modern quenching process is usually realised by water spraying, whereas in the past the quenching process was achieved by immersion. The quenching technique by spraying offers the advantage of on time control as well as pressure regulation [5]. Quenching is a multi-physics process which involves not only heat transfer, but also phase transformation and stress evolution problems. Because of the process complexity, coupled and nonlinear type of the problem, no analytical solution was reported [7].

In this work was studied the structural stress due to solid thermal effects during the 6061 aluminium alloy plates quenching process by water spraying. The phase transformations that occur during quenching have not been taken into consideration in this study. The thermal stresses are generated in the quenching process due to large temperature gradients.

The main challenge for this problem is to define the boundary conditions, respectively the quantitative description of thermo-physical effects occurring on the plate surface during quenching [6].

Numerous researches studies have been conducted in last three decades to develop numerical simulation methods for the prediction of the final state of the material after quenching [12].

Considering the data of the problem, a new approach to the implementation of the boundary conditions was achieved by using the ANSYS Discovery AIM 19.2 modelling software.

\section{The mathematical model}

The quenching process can be characterized as a transient heat conduction problem with convective and radiation boundary conditions. The model object was a 6061 aluminium alloy plate with the dimensions $100 \times 1500$ x $3000 \mathrm{~mm}$ (Figure 1).

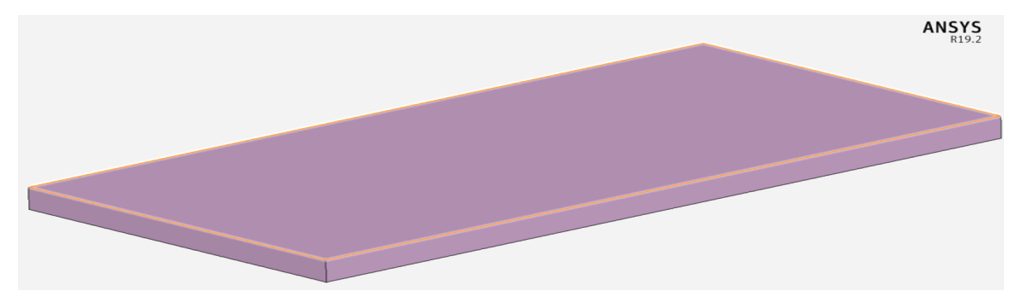

Figure 1. The computational domain for the 6061 aluminium alloy plate

The tests and measurements were performed on the independent equipment for the research of the aluminium alloy quenching process (Figures 2 - 3), recently commissioning into the R\&D Department of ALRO and provided with the latest technology.

At $t=0 \mathrm{~s}$, the plate was assumed to be entirely in the solution heat treatment area (Figure 4). At this moment, the plate temperature is assumed to be $550 \check{\mathrm{r} C}$, taking into consideration the temperature set points for this treatment.

Depending on the thickness of the 6061 aluminium alloy plates to be treated, they are subjected to the solution treatment at $550 \check{\mathrm{r}} \mathrm{C}$, but for different soaking time, as this parameter is directly dependent on the plate thickness. The solution temperature depends on the alloy chemical composition and desired aging extent. The solidus temperature is recorded at 582 


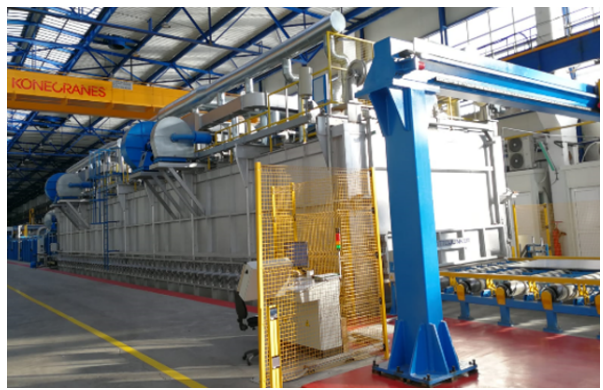

Figure 2. The independent equipment for the research of aluminium alloy quenching process.

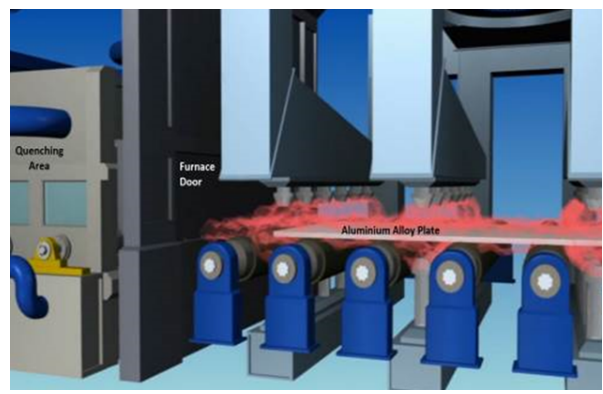

Figure 4. The plate is in the furnace.

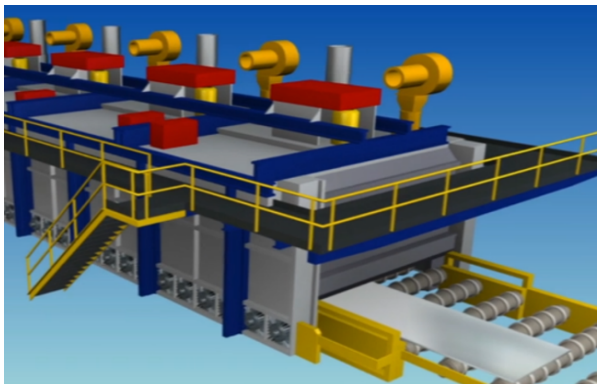

Figure 3. The plate is ready for solution heat treatment.

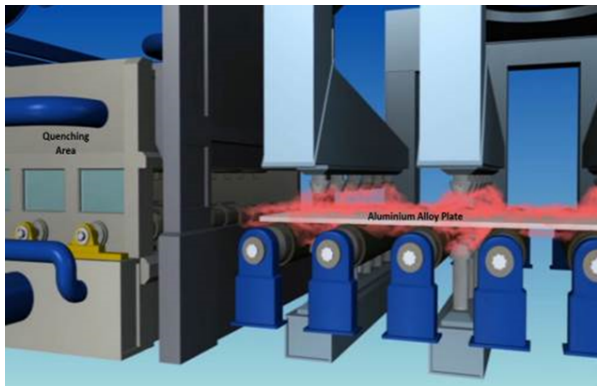

Figure 5. The furnace door is opened and the plate is ready to enter in the quenching area.

r̆C for 6061 aluminium alloy [17]. and the soaking time and temperature are specified in standards. The soaking time may however differ from one standard to another.

The furnace door practically opens and the plate exits from the furnace by the movement induced by the conveying rollers (Figure 5). The plate speed is a specific parameter to each quenching recipe, and is dependent on the type of alloy and the thickness of the plate.

At $\mathrm{t}=1 \mathrm{~s}$, the plate was assumed to come in direct contact with the cooling medium, respectively demineralized water in our case. The demineralized water temperature is controlled and it is usually in the range of 2535 řC. Depending on the length of the plate, after several seconds, the plate will be entirely in the quenching area (Figure 6). Next, the plate will enter in the post-quenching area (Figure 7).

According to the quenching recipe used for the 6061 aluminium alloy plate, $100 \mathrm{~mm}$ thickness, the plate advancing speed in the quenching area is $125 \mathrm{~mm} / \mathrm{s}$. 25 seconds after the furnace door is opened, the plate will be completely in the quenching area. In our case, the length of the quenching area is $3000 \mathrm{~mm}$, therefore, after 24 seconds the plate will be completely out of the quenching area. The simulation of the quenching process will be carried out for a period of 49 seconds, from the moment the plate exits the furnace until it entries completely in the after quenching area.

The 6061 aluminium alloy plate used in our model was obtained by hot rolling from a cast slab. After being hot rolled, the 6061 aluminium alloy plates were solution heat treated at $550{ }^{\circ} \mathrm{C}$ and quenched by spray cooling in an industrial facility (Figure 2). The properties 


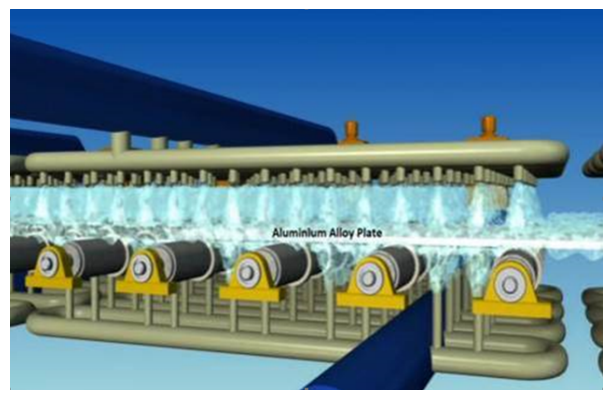

Figure 6. The plate is in the quenching area.

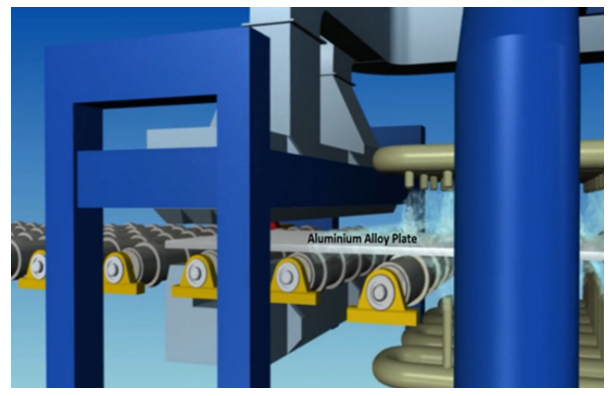

Figure 7. The plate comes out of the quenching area.

of the 6061 aluminium alloy plate used as input data in our model are presented in the table 1.

Table 1. Material properties of the 6061 aluminium alloy, where $\mathrm{T}$ is temperature.

\begin{tabular}{lr}
\hline Density, $\rho\left[\mathrm{kg} / \mathrm{m}^{3}\right]$ & 2700 \\
\hline Youngs modulus,, $\mathrm{E}[\mathrm{GPa}]$ & 69 \\
\hline Poissons ratio, $v$ & 0.33 \\
\hline Tensile yield strength, $\mathrm{S}_{\mathrm{yt}}[\mathrm{MPa}]$ & 275 \\
\hline Tensile ultimate strength, $\mathrm{S}_{\mathrm{ut}}[\mathrm{MPa}]$ & 310 \\
\hline Coefficient of thermal expansion, $\alpha\left[1 /{ }^{\circ} \mathrm{C}\right]$ & $2.51 \mathrm{e}-05$ \\
\hline Heat capacity, $\mathrm{C}_{p}\left[\mathrm{~J} /\left(\mathrm{Kgu}{ }^{\circ} \mathrm{C}\right)\right]$ & $0.0004 \mathrm{u}^{2}{ }^{2}+0.1474 \mathrm{u} T+914.7$ \\
\hline Thermal conductivity, $k,\left[\mathrm{~W} / \mathrm{mu}^{\circ} \mathrm{C}\right]$ & $-0.0002 \mathrm{u}^{2}{ }^{2}+0.115 \mathrm{o} \mathrm{T}+166.44$ \\
\hline
\end{tabular}

It is possible in ANSYS software to define a new material or select it from the software library [2]. The 6061 aluminium alloy was defined as a new material and the data were collected from standards and academic literature. The data concerning the heat capacity and thermal conductivity was taken from the academic literature [13] and interpolated by using the least square method [15]. The other data was taken from the standard [17].

The mathematical equation for the quenching problem was formulated by French physicist and mathematician Joseph Fourier, more than 200 years ago. The most common form of this equation is:

$$
\alpha C_{p} \frac{\partial T}{\partial t}+\nabla(-k \nabla T)=Q
$$

where: $T$ temperature $\left[{ }^{\circ} \mathrm{C}\right], \rho$ density $\left[\mathrm{kg} / \mathrm{m}^{3}\right], C_{p}$ heat capacity $[\mathrm{J} /(K g \cdot \breve{\mathrm{r}} \mathrm{C})], k$ thermal conductivity $[\mathrm{W} /(m \cdot \check{\mathrm{r}} \mathrm{C})], Q$ heat source and $t$ time $(\mathrm{s})$.

By choosing the type of problem, ANSYS has already implemented the main equation(s) of the model, in our case this is equation (1). In fact, defining the mathematical model is reduced to defining the boundary conditions. For transient problems, it is also necessary to define the initial conditions and for the heat problem, the initial condition is:

$$
T_{\text {ini }}=550^{\circ} \mathrm{C}, \text { when } t=0 s \text {. }
$$


The boundary condition necessary for the quenching problem, must be as follows:

$$
-\boldsymbol{n} \cdot(-k \nabla T)=q(A, t)
$$

where $A$ is a point on the plate's surface with the spatial coordinates: $x, y$ and $z$.

Due to existing limitations in ANSYS Discovery AIM 19.2, such a condition can not be implemented. ANSYS gives us the following possibilities for implementing the condition no. (3): inserting a constant into the right-hand side or using a tabular form. It was preferable to use a tabular form due to the better accuracy that can be obtained. A trick was also used, namely to divide the plate volume into smaller volumes taking into account that the plate length is $3000 \mathrm{~mm}$ and the plate speed is $125 \mathrm{~mm} / \mathrm{sec}$, therefore the volume was divided into 24 equal volumes (Figure 8). By realizing this division, it is possible to control the speed of the plate, by simulating different scenarios with various divisions widths.

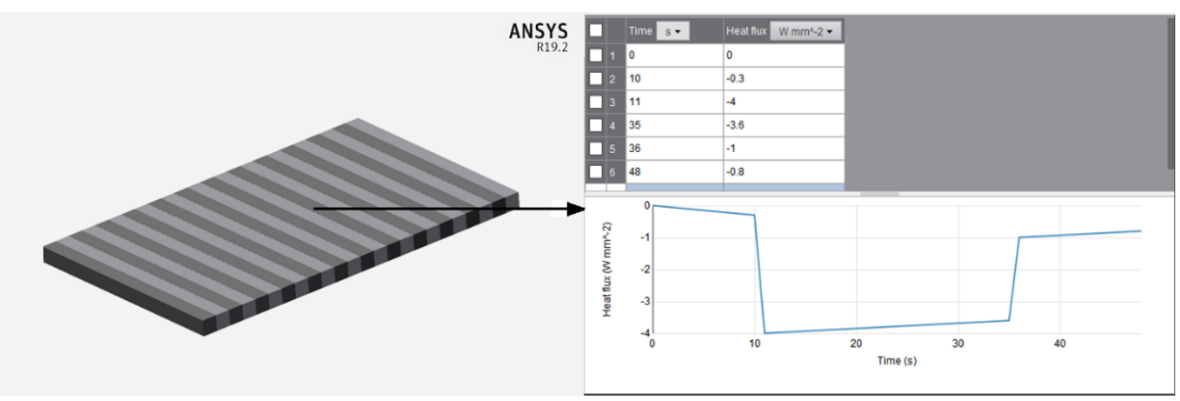

Figure 8. The boundary condition for the $10^{\text {th }}$ volume of the 6061 aluminium alloy plate.

For example, the $10^{\text {th }}$ volume of the plate is not in direct contact with the water in the first 10 seconds, as it is still in the furnace, so the lost heat is very low. But starting the $11^{\text {th }}$ second, the $10^{\text {th }}$ volume of the plate comes in direct contact with the cooling medium and the lost heat increases considerably until the plate exits from the quenching area, respectively in the $35^{\text {th }}$ second. Heat flux values were established following the experiments and tests indicated in Section 3.1. The heat flux, for the outside surfaces of the $10^{\text {th }}$ volume, is presented graphically in Figure 8.

The thermal strain $\left(\varepsilon_{t h}\right)$ depends on the temperature, $T$, the stress-free initial temperature, $T_{\text {ini }}$, and the thermal expansion vector, $a$ :

$$
\varepsilon_{t h}=\alpha\left(T-T_{i n i}\right)
$$

It was assumed that the phase transition does not change during the quenching process. Since the rolling before quenching has little effect on the quenching residual stress $[10,16]$, the zero stress state is considered as the initial state.

The strain consists of elastic $\left(\varepsilon_{e l}\right)$ and thermal $\left(\varepsilon_{t h}\right)$ contributions so that:

$$
\varepsilon=\varepsilon_{e l}+\varepsilon_{t h}
$$

The stress is related to the strains by:

$$
\sigma=E \varepsilon
$$

For the structural model, the boundary conditions are of , free (unconstrained) type, except for the four corners (points) on the bottom of the plate, where they are, fixed on the transversal direction of the plate and free" in the plane area (Figure 9). 


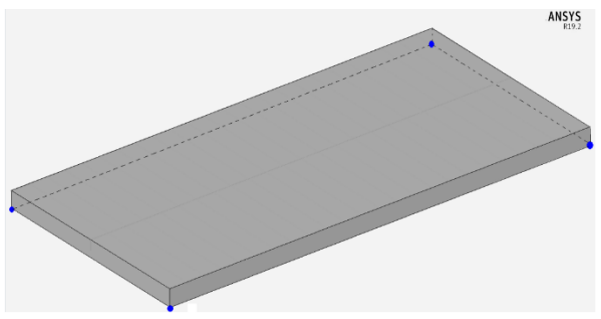

Figure 9. The boundary condition for the $10^{\text {th }}$ volume of the 6061 aluminium alloy plate.

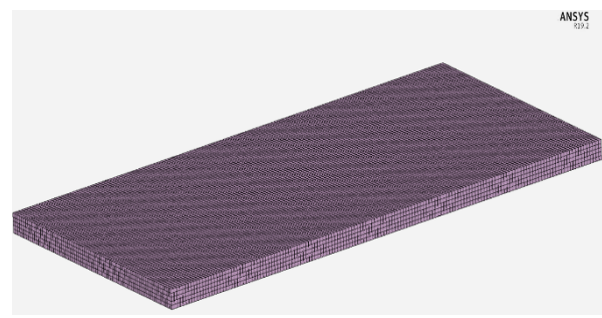

Figure 10. FEM mesh for aluminium alloy plate (approx. 200,000 hexahedral elements).

The temperature value arising from the heat problem will be used as the input data in the structural problem for calculating the structural stress due to solid thermal effects on the studied aluminium alloy plate.

The hexahedral elements were used for the mesh operation (Figure 10).

The distributed sparse direct solver was selected to solve the mathematical model. This solver decomposes a large sparse matrix into smaller sub matrices, and then sends these sub matrices to multiple cores [8].

\section{Numerical simulation results. Discussion}

All the simulations were run using the commercial finite element package ANSYS Discovery AIM 19.2. Elapsed time spent computing solution was around 10000 seconds and a PC Intel(R) Core(TM) i7-6700 CPU @ 3.40GHz was used.

\subsection{Temperature evolution during the quenching process}

The industrial experiments were a very important part in determining the heat flux that is lost during quenching, aiming to obtain a higher accuracy between the measured and the calculated temperature in the centre of the plate.

Industrial experiments were achieved for the validation of the numerical results. For this, a 6061 aluminium alloy plate with dimensions 100x1500x3000 mm was considered, in which the $\mathrm{K}$ type thermocouples were inserted at different depths: $2 \mathrm{~mm}, 50 \mathrm{~mm}$ and $98 \mathrm{~mm}$ (Figures 11 12). By using a Datapaq Tpaq2116A recorder system, the data were automatically collected and saved as an electronic record.

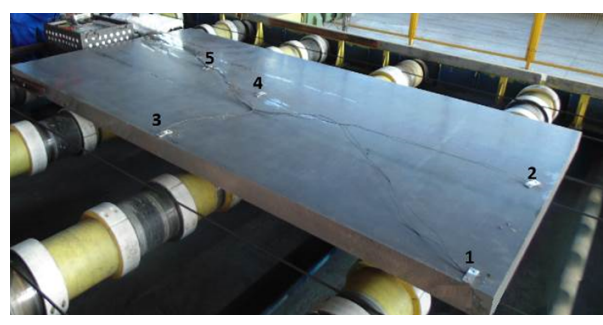

Figure 11. Positioning of the thermocouples during the quenching tests.

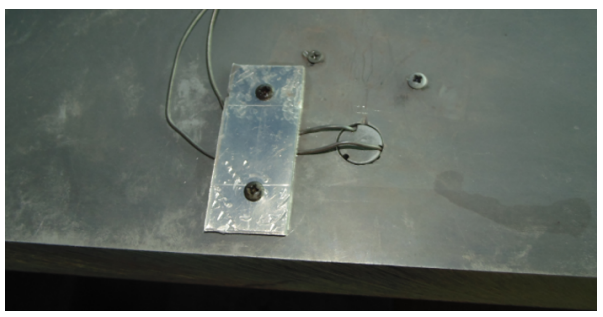

Figure 12. Detail with the position of thermocouple. 
The temperature evolution during the quenching process for different times is presented in the Figures $13-18$.

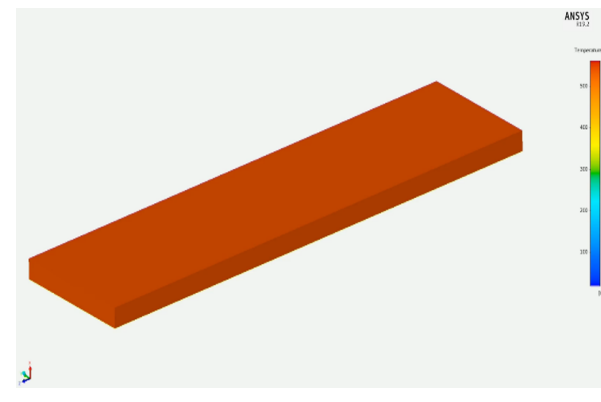

Figure 13. $t=0 \mathrm{~s}$.

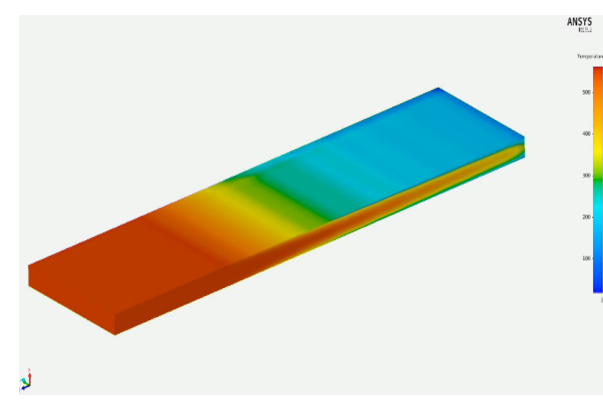

Figure 15. $t=16 \mathrm{~s}$.

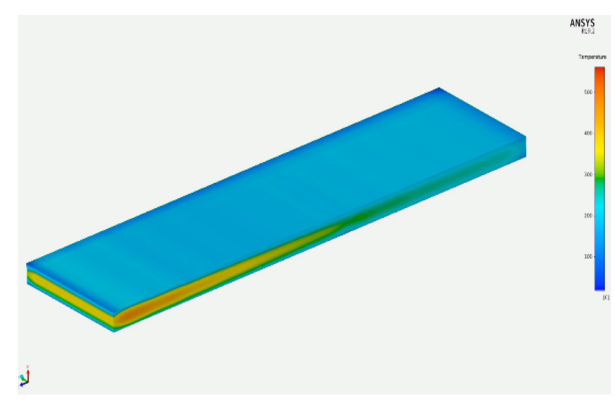

Figure 17. $t=32 \mathrm{~s}$.

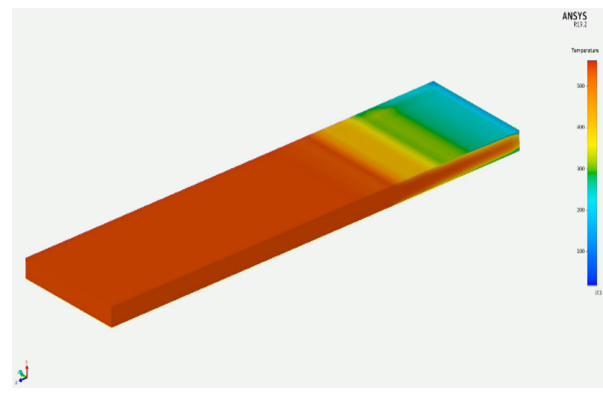

Figure 14. $t=8 \mathrm{~s}$.

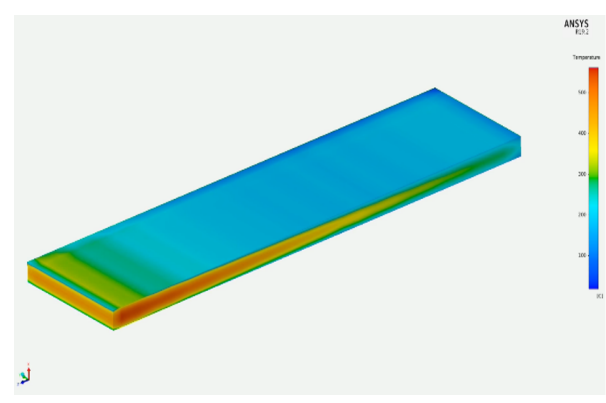

Figure 16. $t=24 \mathrm{~s}$.

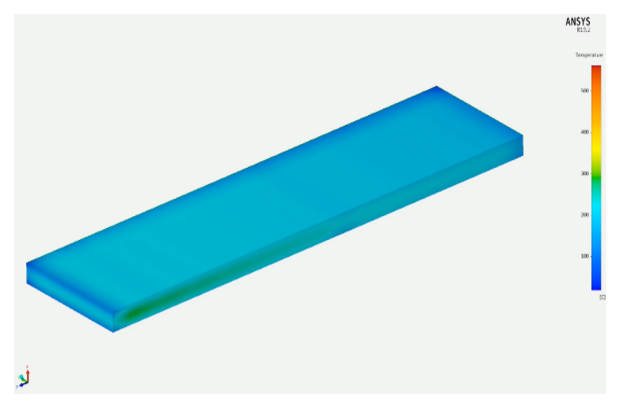

Figure 18. $t=48 \mathrm{~s}$. 
The quenching rate performance of the plate can be seen from the temperature evolution in the centre of the plate during this process. Figure 19 shows the evolution of this temperature, measured vs. numerical values.

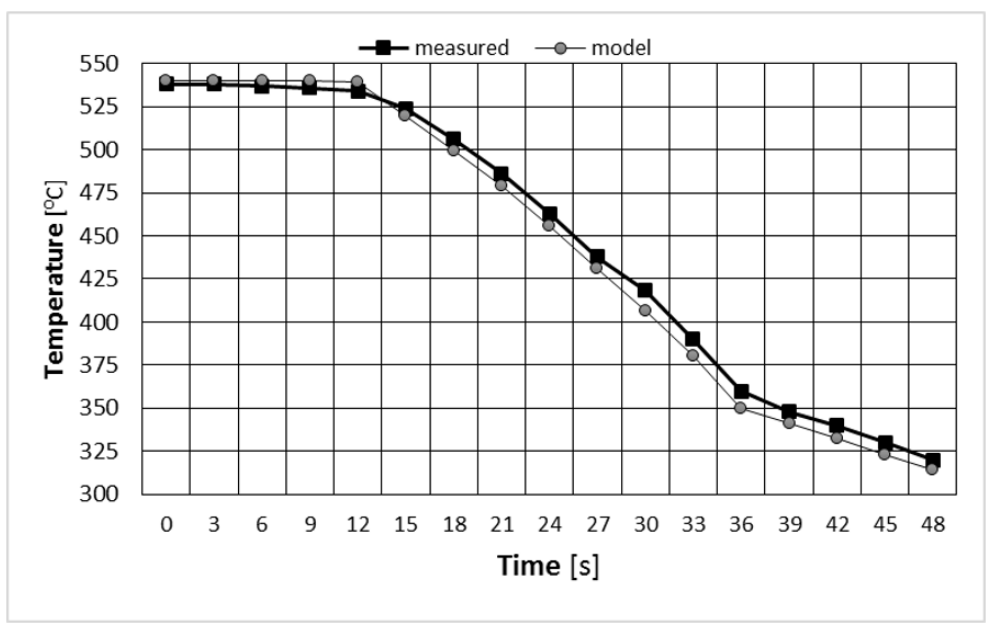

Figure 19. Evolution of temperature in the centre of the plate during the quenching process.

The measurements were saved within a range of 3 seconds. Minor differences between the measured values and the calculated ones were observed. The highest difference is $11{ }^{\circ} \mathrm{C}$, at 30 seconds after the quenching process started. It must also be taken into consideration the measurement error of the thermocouples that are being used, respectively s $4{ }^{\circ} \mathrm{C}$.

\subsection{Structural stress due to solid thermal effects}

A plate deformation of $102.7 \mathrm{~mm}$ was determined from the structural model (Figure 20).

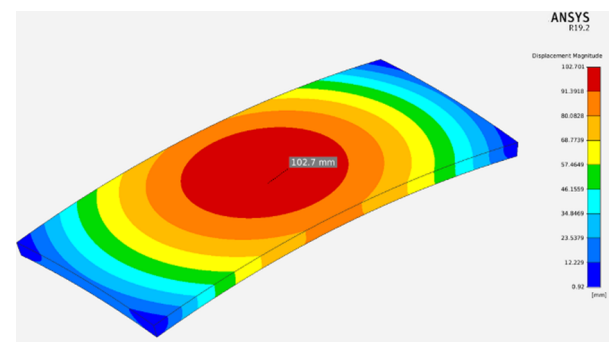

Figure 20. Deformation of the 6061 aluminium alloy plate after the quenching process.

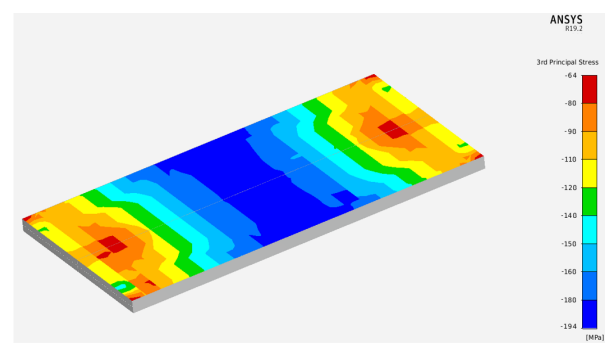

Figure 21. The residual stress on the surface of the 6061 aluminium alloy plate numerical values.

The residual stresses on the plate surface immediately after the quenching is represented in Figure 21. Residual stresses were determined by calculation of the Third principal stresses (the most negative or compressive). Accordingly, the plate deformation involves a similar stresses distribution on the plate surface. 


\section{Conclusions}

The mathematical model of the water spraying quenching process was presented and implemented using the ANSYS Discovery AIM 19.2 software and validated on the recently commissioned industrial equipment provided with the latest technology in the field.

The transient heat transfer problem between the cooling medium (demineralized water) and the 6061 aluminum alloy plate was solved and the temperature evolution was obtained as a function of time and position within the plate. The differences between the measured and the numerical temperature values were very small, of maximum $11^{\circ} \mathrm{C}$.

For the structural analysis, the temperature of the heat transfer problem was used as input data. The obtained numerical results presented the correlation between the deformation and the residual stress of the plate.

The findings are useful to better understand the stresses generated by thermal effects during the quenching process, and to control and minimize them by adjusting the quenching parameters.

\section{Acknowledgement}

Part of the cost of the industrial equipment used to obtain the results presented in this work was funded by European Union through Competitiveness Operational Programme, Priority Axis 1 Research, Technological Development and Innovation, within the project Investments in the RED Department of ALRO aiming at improving the research infrastructure for the aluminium alloy heat treated plates with high qualification industrial applications, based on the Funding Contract no. 42/05.09.2016.

\section{References}

[1] M. Belte and D. Drăgulin, ATC Aluvation, Importance of the cooling rate during the heat treatment process of aluminium, International Aluminium Journal, 61-64 (2018).

[2] X. Chen and Y. Liu, Finite Element Modeling and Simulation with ANSYS Workbench (A CRC Press, August 11, 2014).

[3] T. Croucher, Quenching of aluminum alloys: what this key step accomplishes, Heat Treating 14(5) May, 20-21 (1982).

[4] F. Fracasso, Influence of quench rate on the hardness obtained after artificial ageing of an Al-Si-Mg alloy, (Master Thesis, University of Padova, Padova, Italy, 2010).

[5] R. Jeschar, E. Specht, and C.H.R. Köhler, Chapter 6 - Heat Transfer during Cooling of Heated Metals with Vaporizable Liquids, Quenching Theory and Technology, Second Edition (Boidar Licic, CRC Press, Boca Raton, FL, 2010) 158-178.

[6] J. Lemaitre, Handbook of Materials Behavior Models (Academic Press, San Diego, CA, 2001) $1200 \mathrm{pp}$.

[7] J. Mackerle, Finite element analysis and simulation of quenching and other heat treatment processes: A bibliography (1976-2001), Computational Materials Science 27(3), 313-332 (2003).

[8] S. Moaveni, Finite Element Analysis: Theory and Application with ANSYS (Global Edition, 2014) $936 \mathrm{pp}$.

[9] M.B. Prime and M.R. Hill, Residual stress, stress relief, and inhomogeneity in aluminum plate, Scripta Materialia, 46(1), 77-82 (2002).

[10] P. Rashed and M.M.A. Hossain, Control of Distortion in Aluminium Heat Treatment, Chapter 13, Fundamentals of Aluminium Metallurgy, Recent Advances (Woodhead Publishing Series in Metals and Surface Engineering, 2018) 495-524. 
[11] J.S. Robinson, D.A. Tanner, and C.E. Truman, The origin and management of residual stress in heat-treatable aluminium alloys, Strain 50(3), 185-207 (2014).

[12] B.D. Storey, Fluid dynamics and heat transfer, An introduction to the fundamentals (Olin College, 2015).

[13] P.T. Summers, Y. Chen, C.M. Rippe, B. Allen, A.P. Mouritz, S.W. Case, and B.Y. Lattimer, Overview of aluminum alloy mechanical properties during and after fires, Fire Science Reviews 4(3) (2015).

[14] G.E. Totten, G.M. Webster, and C.E. Bates, Chapter 20 - Quenching, Handbook of Aluminum - Volume 1: Physical Metallurgy and Processes (Eds. G.E. Totten,and D.S. MacKenzie, CRC Press, Boca Raton, Fla., 2003) 971-1062.

[15] J. Wolberg, Data Analysis Using the Method of Least Squares: Extracting the Most Information from Experiments (Springer-Verlag, New York, 2005) 263 pp.

[16] P.J. Withers, H. Bhadeshia, Residual stress-II: nature and origins, Mater. Sci. Tech. 17, 366-375 (2001).

[17] ASM Handbook Volume 2: Properties and Selection: Nonferrous Alloys and SpecialPurpose Materials (ASM International, 1990) 1328 pp. 